Accepted for publication in ApJL

\title{
Testing the External Shock Model of Gamma-Ray Bursts using the Late-Time Simultaneous Optical and X-ray Afterglows
}

\author{
Yuji Urata ${ }^{1}$, Ryo Yamazaki ${ }^{2}$, Takanori Sakamoto ${ }^{3}$, Kuiyun Huang $^{4}$, \\ Weikang Zheng ${ }^{5}$, Goro SATO $^{3}$, Tsutomu Aoki ${ }^{6}$, Jinsong DenG ${ }^{5}$, Kunihito IokA ${ }^{7}$, \\ WingHuen $\mathrm{IP}^{8}$, Koji S. Kawabata ${ }^{9}$, YiHsi $\mathrm{LEE}^{8}$, Xin $\mathrm{LiPInG}^{5}$, Hiroyuki Mito ${ }^{6}$, \\ Takashi MiYAta ${ }^{6}$, Yoshikazu NAKAdA ${ }^{6}$, Takashi OHsugi ${ }^{2,9}$, Yulei QIU ${ }^{5}$, Takao SoyAno ${ }^{6}$, \\ Kenichi Tarusawa ${ }^{6}$, Makoto TAshiro ${ }^{1}$, Makoto Uemura ${ }^{9}$, Jianyan $\mathrm{WeI}^{5}$, and \\ Takuya YAmashita ${ }^{8}$
}

\begin{abstract}
We study the "normal" decay phase of the X-ray afterglows of gamma-ray bursts (GRBs), which follows the shallow decay phase, using the events simultaneously observed in the R-band. The classical external shock model - in which neither the delayed energy injection nor time-dependency of shock micro-physics is considered - shows that the decay indices of the X-ray and R-band light curves, $\alpha_{\mathrm{X}}$ and $\alpha_{\mathrm{O}}$, obey a certain relation, and that in particular, $\alpha_{\mathrm{O}}-\alpha_{\mathrm{X}}$ should be larger than $-1 / 4$ unless the ambient density increases with the distance from the central engine. For our selected 14 samples, we have found that 4 events violate the limit at more than the $3 \sigma$ level, so that a fraction of events are outliers of the classical external shock model at the "normal" decay phase.
\end{abstract}

\footnotetext{
${ }^{1}$ Department of Physics, Saitama University, Shimo-Okubo, Saitama, 338-8570, Japan; urata@heal.phy.saitama-u.ac.jp

${ }^{2}$ Department of Physics, Hiroshima University, Higashi-Hiroshima, Hiroshima 739-8526, Japan; ryo@theo.phys.sci.hiroshima-u.ac.jp

${ }^{3}$ NASA Goddard Space Flight Center, Greenbelt, MD 20771, USA

${ }^{4}$ Academia Sinica Institute of Astronomy and Astrophysics, Taipei 106, Taiwan, Republic of China

${ }^{5}$ National Astronomical Observatories, Chinese Academy of Sciences, Beijing 100012, China

${ }^{6}$ Kiso Observatory, Institute of Astronomy, The University of Tokyo, Kiso-muchi, Kiso-gun, Nagano 397-0101, Japan

${ }^{7}$ Departments of Physics, Kyoto University, Kitashirakawa, Sakyo-ku, Kyoto 606-8602, Japan

${ }^{8}$ Institute of Astronomy, National Central University, Chung-Li 32054, Taiwan, Republic of China

${ }^{9}$ Astrophysical Science Center, Hiroshima University, Higashi-Hiroshima, Hiroshima 739-8526, Japan
} 
Subject headings: gamma rays: bursts — gamma rays: observation

\section{Introduction}

Gamma-ray bursts (GRBs) consist of two phases: prompt GRB emission and subsequent afterglows. How long the prompt GRB emission lasts and when the transition from the prompt GRB to the afterglow occurs have been long-standing problems. These problems are tightly related to the mechanism of the central engine of GRBs. The Swift satellite has brought us early, dense, and detailed data on the afterglows of GRBs in various observation bands. Now, we are entering the era of multi-wavelength observations; especially optical and X-ray bands, which tell us some hints for answering the problems.

Contrary to the expectation in the pre-Swift era, Swift X-Ray Telescope (XRT) data have revealed complex temporal behavior of the X-ray afterglow (Burrows et al. 2005; Tagliaferri et al. 2005; Nousek et al. 2006; O'Brien et al. 2006a; Willingale et al. 2007). Initially, it decays very steeply, whose most popular interpretation is the tail emission of the prompt GRB (Kumar \& Panaitescu 2000; Zhang et al. 2006; Yamazaki et al. 2006), although other possibilities have been proposed (e.g., Zhang et al. 2007). At several hundreds of seconds after the burst trigger, the shallow decay phase begins until $\sim 10^{4} \mathrm{sec}$, whose origin is quite uncertain (e.g., Toma et al. 2006; Ioka et al. 2006; Zhang 2007). After the shallow decay phase ends, the X-rays subsequently decays with the decay index usually steeper than unity, which was expected in the pre-Swift era. This decay behavior can be well explained by the classical external shock model (Sari et al. 1998), in which neither the delayed energy injection nor time-dependency of shock micro-physics is considered. Hence this phase is sometimes called the normal decay phase.

However, as the number of the X-ray observations increases, it is getting suspicious that the normal decay phase arises from the external shock. In the steep and the shallow decay phases, the X-ray light curves sometimes possess large bumps, called the X-ray flares (Chincarini et al. 2007; Falcone et al. 2007), and/or dips that cannot be explained by the external shock model (Ioka et al. 2005). Furthermore, for an extreme example, GRB 070110 showed a rather complex X-ray afterglow with a sudden drop at $\sim 2 \times 10^{4}$ sec after the burst trigger as the end of the shallow decay phase (Troja et al. 2007). These observational facts may tell us that the steep and the shallow decay phases are likely due to late internal dissipation of the energy produced by the long-acting central engine. On the other hand, the

X-ray spectrum remains unchanged across the shallow-to-normal transition (Nousek et al. 2006), which may imply that the shallow and the normal decay phases are of the same origin. Therefore, it might be that the normal phase comes from the internal energy dissipation. 
The observed optical afterglow is also complicated and in an early epoch $\left(\lesssim 10^{3} \mathrm{sec}\right)$, there is a diversity (Zhang 2007; Doi et al. 2007). On the other hand, it was found in the preSwift era that for almost all events, the behavior at $\gtrsim 0.1$ day after the burst could be well

explained by the classical external shock model (e.g., Panaitescu \& Kumar 2001; Urata et al. 2003), although some events showed complex light curves with dips and/or bumps (e.g., Holland et al. 2003; Lipkin et al. 2004; Urata et al. 2007b). This epoch corresponds to the normal decay phase of the X-ray afterglow. Those previous studies are mainly based on the optical bands, because the X-ray observation was sparse at that time. In the Swift era, we are starting to have the simultaneous optical and X-ray afterglow data in the epoch $\gtrsim 0.1$ day after the burst thanks to the rapid and the dense X-ray observation by the XRT.

In this Letter, we study the normal decay phase of the X-ray afterglows simultaneously observed in optical R-bands, and investigate whether it is consistent with the classical external shock model or not. We perform a simple test using the optical and the X-ray decay indices, $\alpha_{\mathrm{O}}$ and $\alpha_{\mathrm{X}}$, where we use a notation, $F_{\nu} \propto t^{-\alpha} \nu^{-\beta}$. For example, in the classical external shock model with uniform ISM, they are related to the power-law index of the electron distribution, $p(>2)$, as $\alpha_{\mathrm{O}}=3(p-1) / 4$ and $\alpha_{\mathrm{X}}=(3 p-2) / 4$, respectively, since the cooling frequency $\nu_{\mathrm{c}}$ usually lies between the optical and X-ray bands (Sari et al. 1998). Eliminating $p$, we obtain $\alpha_{\mathrm{O}}-\alpha_{\mathrm{X}}=-1 / 4$. Similarly, for the wind environment, we derive $\alpha_{\mathrm{O}}-\alpha_{\mathrm{X}}=1 / 4$ (Chevarier \& $\mathrm{Li}$ 2000). These relations between $\alpha_{\mathrm{O}}$ and $\alpha_{\mathrm{X}}$ are also valid in the case of $1<p<2$ (Dai \& Cheng 2001). Therefore, through the relation between $\alpha_{\mathrm{O}}$ and $\alpha_{\mathrm{X}}$, one can test the classical external shock model. In the pre-Swift era, similar study has been done for BeppoSAX GRBs (De Pasquale et al. 2006b). However, compared with the Swift GRBs, their X-ray data were not well enough to identify the normal decay phase and to determine the decay index with small uncertainties. We can now obtain more dense X-ray and optical data and can determine $\alpha_{\mathrm{O}}$ and $\alpha_{\mathrm{X}}$ with much less ambiguity. Finally it is noted that in this Letter, we do not consider the spectral indices, $\beta_{\mathrm{O}}$ and $\beta_{\mathrm{X}}$, because they have at present large uncertainties; $\beta_{\mathrm{O}}$ fairly depends on the assumed dust model, and the low X-ray flux at the epoch we are interested in makes us difficult to constrain $\beta_{\mathrm{X}}$ with precision which we need to test the model.

\section{Decay indices of X-ray and R-band afterglow in the normal decay phase}

We consider long GRBs that are followed-up by Swift XRT from the beginning of 2005 to the end of 2006. The Swift XRT data are systematically analyzed using our pipeline script. The cleaned event data of the Window Timing (WT) and the Photon Counting (PC) mode from the Swift Science Data Center (SDC) are used in the whole process. Although both WT 
and PC mode data are processed in the pipeline, hereafter, we are only focusing on the process of the PC mode data. The search of the X-ray afterglow counterpart, a construction of the $\mathrm{X}$-ray light curve, and a fitting process of the X-ray light curve and spectra are performed automatically using the standard XRT softwares and calibration database (HEASoft 6.2 and CALDB 20070531). The source region is selected as a circle of $47^{\prime \prime}$ radius. The background region is an annulus of an outer radius of $150^{\prime \prime}$ and an inner radius of $70^{\prime \prime}$ excluding the background X-ray sources detected by ximage in circle region of $47^{\prime \prime}$ radius. The light curve is binned based on the number of photons required to meet at least $5 \sigma$ (Sakamoto et al. 2007). We select the samples of the X-ray afterglows which have a smooth transition from the shallow to the normal decay phases at $\gtrsim 10^{3}$ sec. Samples with X-ray flares have been excluded. Then, we find the start time of the normal decay phase of the X-ray afterglow $\left(\alpha_{\mathrm{X}} \gtrsim 1\right)$, and extract events in which well-sampled R-band light curves are available during the normal decay phase.

The light curve data in the R-band are published in literatures or observed by the East Asian GRB Follow-up Network (EAFON; Urata et al. 2005)11 and the KANATA telescope. The R-band data taken by us are processed as in the following. A standard routine, including bias subtraction, dark subtraction, and flat-fielding corrections with appropriate calibration data is employed to process the data using IRAF. Flux calibrations are performed using the APPHOT package in IRAF, referring to the standard stars suggested by Landolt (1992). For each data set, the one-dimensional aperture size is set to 4 times as large as the full-width at half maximum of the objects. The magnitude of error for each optical image is estimated as $\sigma_{\mathrm{e}}^{2}=\sigma_{\mathrm{ph}}^{2}+\sigma_{\mathrm{sys}}^{2}$, where $\sigma_{\mathrm{ph}}$ represents the photometric errors for each afterglow, estimated from the output of IRAF PHOT, and $\sigma_{\text {sys }}$ is the photometric calibration error estimated by comparing our instrumental magnitudes. When we combine data which are obtained at several different sites, we re-calibrate each data set by our photometric manner (e.g., Huang et al. 2007; Urata et al. 2007a, 2003). These efforts decrease systematic differences and yield realistic light curves.

There are 14 GRBs which have a good coverage with both X-ray and optical bands at the normal decay phase. Among them, optical data of 11 events have been already published in literatures. For unpublished data obtained by EAFON, detailed light curves in the X-ray and the optical bands are presented elsewhere (Urata et al. 2007, in preparation). For those samples, we identify the normal decay phase that is well described by a single power-law decay model and derive $\alpha_{\mathrm{X}}$. During the phase, we find that the optical light curves are well fitted with a single power-law model in the time interval shown in Table11in which the decay

\footnotetext{
${ }^{1}$ In this paper, the samples are mainly taken using Kiso $1.05 \mathrm{~m}$ Schmited telescope(Urata et al. 2003), Lulin One-meter telescope(Huang et al. 2005) and Xinglong 0.8m telescope(Deng et al. 2007).
} 
index, $\alpha_{\mathrm{O}}$, is determined. All results are summarized in Table 1, Figure 1 shows $\alpha_{\mathrm{O}}$ as a function of $\alpha_{\mathrm{X}}$, while Figure 2 shows the value of $\alpha_{\mathrm{O}}-\alpha_{\mathrm{X}}$ for each event. The quoted errors in this Letter are at the $1 \sigma$ confidence level.

\section{Results and Discussion}

Let us consider the case of the minimum frequency $\nu_{\mathrm{m}}$ smaller than the R-band frequency $\nu_{\mathrm{R}}\left(\nu_{\mathrm{m}}<\nu_{\mathrm{R}}\right)$, which is a reasonable assumption for several bright bursts in the pre-Swift era. In this case, the spectral index of the optical afterglow is positive, $\beta_{\mathrm{O}}>0$, which is consistent with the previous observational results (see Table 2 of Kann et al. 2006). The decay and the spectral indices are calculated as shown in Table 2 by the classical external shock model with ambient matter density dependent on the radius, $n \propto r^{-s}$ where we assume $s>0$. Since the Lorentz factor of the relativistically expanding shell evolves with the observer time as $\Gamma \propto t^{-\frac{3-s}{8-2 s}}, s<3$ is needed in order for the shell to decelerate. If $\nu_{\mathrm{m}}<\nu_{\mathrm{R}}<\nu_{\mathrm{c}}<\nu_{\mathrm{X}}$, we derive

$$
\alpha_{\mathrm{O}}-\alpha_{\mathrm{X}}=-\frac{1}{4}+\frac{s}{8-2 s}
$$

which is valid for $1<p<2$ or $2<p$, so that $\alpha_{\mathrm{O}}-\alpha_{\mathrm{X}}$ ranges between $-1 / 4$ and $5 / 4$ if $0<s<3$. For the cases of $\nu_{\mathrm{m}}<\nu_{\mathrm{R}}<\nu_{\mathrm{X}}<\nu_{\mathrm{c}}$ or $\nu_{\mathrm{m}}<\nu_{\mathrm{c}}<\nu_{\mathrm{R}}<\nu_{\mathrm{X}}, \alpha_{\mathrm{O}}-\alpha_{\mathrm{X}}$ should be zero. As can be seen in Fig. 2, among 14 events considered in this paper, 4 events (GRB 050319, 050401, 060206, 060323) are below the line $\alpha_{\mathrm{O}}-\alpha_{\mathrm{X}}=-1 / 4$ at more than the $3 \sigma$ level, so that a fraction of bursts are outliers of the classical external shock model at the normal decay phase. De Pasquale et al. (2006b) have performed similar study and found two out of 12 events have $\alpha_{\mathrm{O}}-\alpha_{\mathrm{X}}$ significantly below $-1 / 4$, which was roughly consistent with our result (see Table 5 of their paper).

Liang et al. (2007) studied the $\alpha_{\mathrm{X}}-\beta_{\mathrm{X}}$ relation of the normal decay phase and found that there are several outliers of the classical external shock model. Their outliers have large $\alpha_{\mathrm{X}}>2$. In our sample, however, outliers of $\alpha_{\mathrm{O}}-\alpha_{\mathrm{X}}$ relation exists even if their $\alpha_{\mathrm{X}}$ of around 1.5, and their $\alpha_{\mathrm{X}}-\beta_{\mathrm{X}}$ relations are consistent with the classical external shock model (see Fig. 5 of Liang et al. 2007). This fact, therefore, strengthens the importance of the multi-wavelength studies at the normal decay phase to test the classical external shock model.

There are several possibilities leading to $\alpha_{\mathrm{O}}-\alpha_{\mathrm{X}}<-1 / 4$. One is to consider $s<0$ case (e.g. Yost et al. 2003). If $s \lesssim-4$, then $\alpha_{\mathrm{O}}-\alpha_{\mathrm{X}} \lesssim-0.5$, however, there is no theoretical reason to consider such a steeply rising profile. Another is to consider the delayed energy injection (Rees \& Mészáros 1998) and/or time-variable shock micro-physics parame- 
ters (Yost et al. 2003). Here, we consider $\nu_{\mathrm{m}}<\nu_{\mathrm{R}}<\nu_{\mathrm{c}}<\nu_{\mathrm{X}}$ for simplicity. The generalized forms of $\alpha_{\mathrm{O}}$ and $\alpha_{\mathrm{X}}$ are then derived by Panaitescu et al. (2006) based on the assumptions $E(>\Gamma) \propto \Gamma^{-e}, \varepsilon_{B} \propto \Gamma^{-b}, \varepsilon_{e} \propto \Gamma^{-i}$, and $n \propto r^{-s}$, where $s<3$. Then, from their derived formula, we obtain

$$
\begin{aligned}
\alpha_{\mathrm{O}}-\alpha_{\mathrm{X}}= & -\frac{1}{4}+\frac{s}{8-2 s} \\
& -\frac{3-s}{4(e+8-2 s)}\left(\frac{4-3 s}{4-s} e+3 b\right),
\end{aligned}
$$

which is independent of $i$ and $p$. We find that $\alpha_{\mathrm{O}}-\alpha_{\mathrm{X}}<-1 / 4$ is achieved if $e+3 b>0$ for the uniform ISM case $(s=0)$, or if $b-e>8 / 3$ for the wind medium case $(s=2)$. Note that these cases have been discussed for the pre-Swift GRBs (Piro et al. 1998; Yost et al. 2003; Corsi et al. 2005).

Another possibility to explain the outliers may be that the X-ray flares superposing on the X-ray afterglow could steep the apparent decay index of the X-ray. X-ray flares are usually more active in the initial phase, so that they may enhance the early X-ray flux. In this case the X-ray flare should not be spiky but relatively smooth, and the late afterglow is just an ordinary afterglow.

Although the external shock model is still viable, the afterglow emissions of outliers may be capable of the internal shock origin. Such a possibility has been proposed by Ghisellini et al. (2007). Then, the optical and X-ray emission in the late phase are of different origins. It is also possible in this model that a chromatic break occurs at $\sim 1$ day after the burst, which was believed to be achromatic in the pre-Swift era and to be caused by the jet collimation effects (Panaitescu et al. 2006; Huang et al. 2007; Sato et al. 2007). Or a cannonball model may account for our outliers (Dado et al. 2007).

We would like to thank Drs. Shiho Kobayashi and Bing Zhang, and the anonymous referee for useful comments. This work was supported in part by Grants-in-Aid for Scientific Research of the Japanese Ministry of Education, Culture, Sports, Science, and Technology 18001842 (Y. U.), 18740153 (R. Y.). This work was also supported by NSC 93-2752-M-008001-PAE and NSC 93-2112-M-008-006. T.S. was supported by an appointment of the NASA Postdoctoral Program at the Goddard Space Flight Center, administered by Oak Ridge Associated Universities through a contract with NASA. This paper was inspired through the discussion during the workshop "Implications of Swift's Discoveries about Gamma-Ray Bursts" at the Aspen Center for Physics. 


\section{REFERENCES}

Antonelli, L. A., et al. 2006, A\&A, 456, 509

Burrows, D. N. et al. 2005, Science, 309, 1833

Cenko, S. B., et al. 2006, ApJ, 652, 490

Chevarier, R. A. \& Li, Z.-Y. 2000, ApJ, 536, 195

Chincarini, G. et al. 2007, astro-ph/0702371

Corsi, A. et al. 2005, A\&A, 438, 829

Dado, S., Dar, A., \& De Rujula, A. 2007, arXiv:0706.0880

Dai, X. et al. 2007, ApJ, 658, 509

Dai, Z.G. \& Cheng, K. S. 2001, ApJ, 558, L109

Deng, J. et al. 2007, Nuovo Cimento B in press

De Pasquale, M., et al. 2006a, MNRAS, 365, 1031

De Pasquale, M., et al. 2006b, A\&A 455, 813

Della Valle, M., et al. 2006, ApJ, 642, L103

Doi, H., Takami, K., \& Yamazaki, R. 2007, ApJ, 659, L95

Falcone, A. D. et al. 2007, arXiv:0706.1564

Ghisellini, G. et al. 2007, ApJ, 658, L75

Halpern, J. P. et al. 2006, GCN Circ., 5847

Holland, S. T. et al. 2003, ApJ, 125, 2291

Huang, K. Y., et al. 2005, ApJ, 628, L93

Huang, K. Y., et al. 2007, ApJ, 654, L25

Ioka, K., Kobayashi, S., \& Zhang, B. 2005, ApJ, 631, 429

Ioka, K. et al. 2006, A\&A, 458, 7

Kann, D. A., Klose, S., \& Zeh, A. 2006, ApJ, 641, 993 
Klotz, A. et al. 2005, A\&A, 439, L35

Kumar, P., \& Panaitescu, A. 2000, ApJ, 541, L51

Landolt, A. U. 1992, AJ, 104, 340

Liang, E. W., Zhang, B. B., Zhang, B. 2007, arXiv:0705.1373

Lipkin, Y. M. et al. 2004, ApJ, 606, 381

Nousek, J. A. et al. 2006, ApJ, 642, 389

O'Brien, P. T. et al. 2006a, ApJ, 647, 1213

Panaitescu, A. \& Kumar, P. 2001, ApJ, 560, L49

Panaitescu, A. et al. 2006, MNRAS, 369, 2059

Piro, L. et al. 1998, A\&A, 331, L41

Rees, M. \& Mészáros, P. 1998, ApJ, 496, L1

Rykoff, E. S., et al. 2006, ApJ, 638, L5

Sakamoto, T., et al. 2007, submitted to ApJ

Sari, R., Piran, T., \& Narayan, R. 1998, ApJ, 497, L17

Sato, G. et al. 2007, ApJ, 657, 359

Sollerman, J., et al. 2007, A\&A, 466,839

Stanek, K. Z., et al. 2007, ApJ, 654, L21

Tagliaferri, G. et al. 2005, Nature, 436, 985

Toma, K. et al. 2006, ApJ, 640, L139

Troja, E. et al. 2007, submitted to ApJ (astro-ph/0702220)

de Ugarte Postigo, A., et al. 2007, A\&A, 462, L57

Uemura, M. et al. 2007, PASJ, submitted

Urata, Y., et al. 2003, ApJ, 595, L21

Urata, Y., et al. 2005, Nuovo Cimento C Geophys. Sp. Phys. C, 28, 775 
Urata, Y., et al. 2007a, ApJ, 655, L81

Urata, Y., et al. 2007b, ApJ, submitted

Willingale, R. et al. 2007, ApJ, 662, 1093

Woźniak, P. R. et al. 2006, ApJ, 642, L99

Yamazaki, R., Toma, K., Ioka, K., Nakamura, T. 2006, MNRAS, 369, 311

Yost, S. A. et al. 2003, ApJ, 597, 459

Yost, S. A. et al. 2007, ApJ, 657, 925

Zhang, B. et al. 2006, ApJ, 642, 354

Zhang, B. 2007, Chin. J. Astron. Astrophys. 7, 1

Zhang, B. B., E. W. Liang, \& Zhang, B. 2007, astro-ph/0612246 


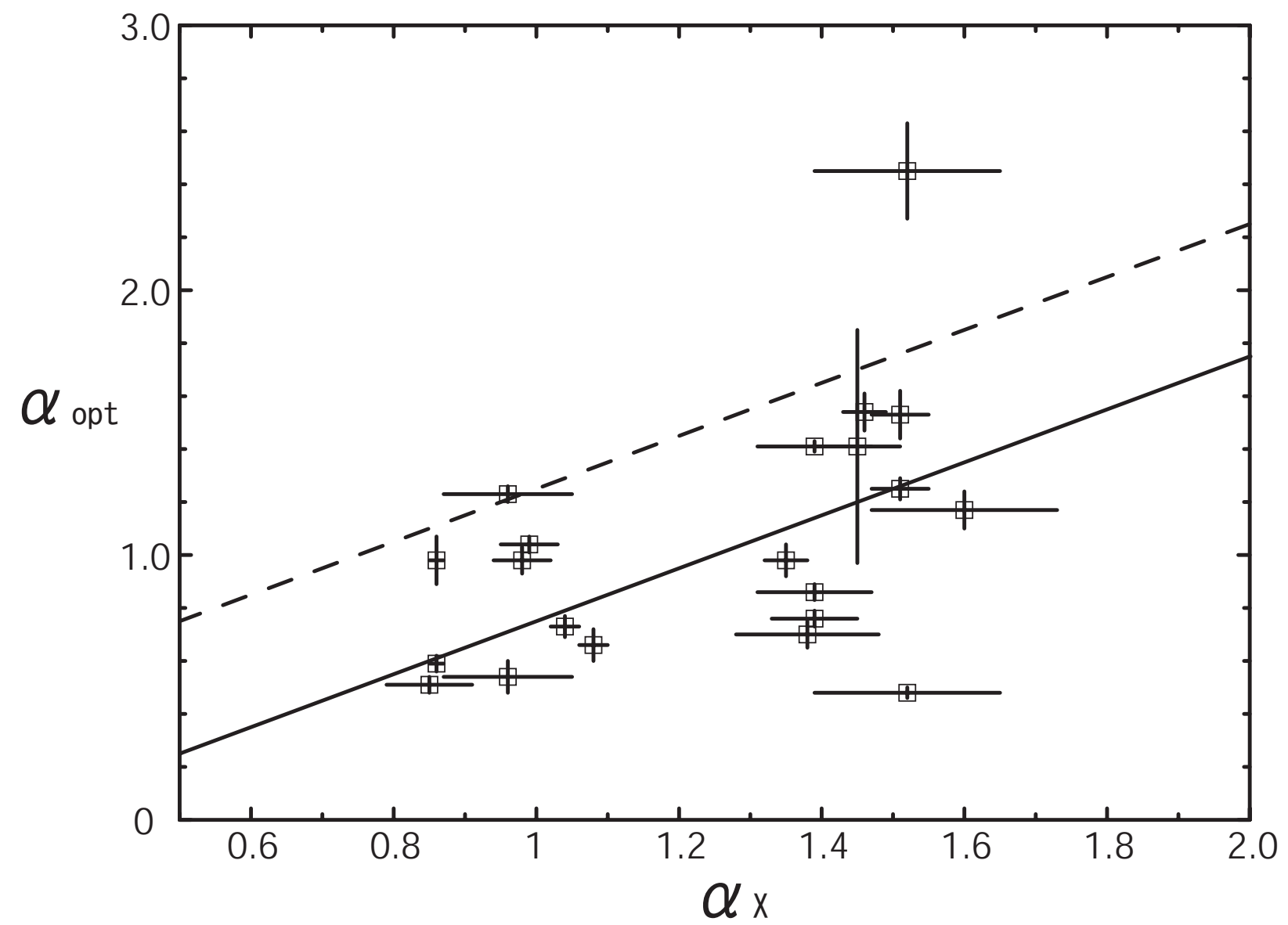

Fig. 1. - The R-band decay index $\alpha_{\mathrm{O}}$ as a function of the X-ray decay index $\alpha_{\mathrm{X}}$ in the normal decay phase. The classical external shock model predicts $\alpha_{\mathrm{O}}-\alpha_{\mathrm{X}}=-1 / 4$ (solid line) and $1 / 4$ (dashed line) for the uniform ISM $(s=0)$ and for the wind medium $(s=2)$ cases, respectively. 


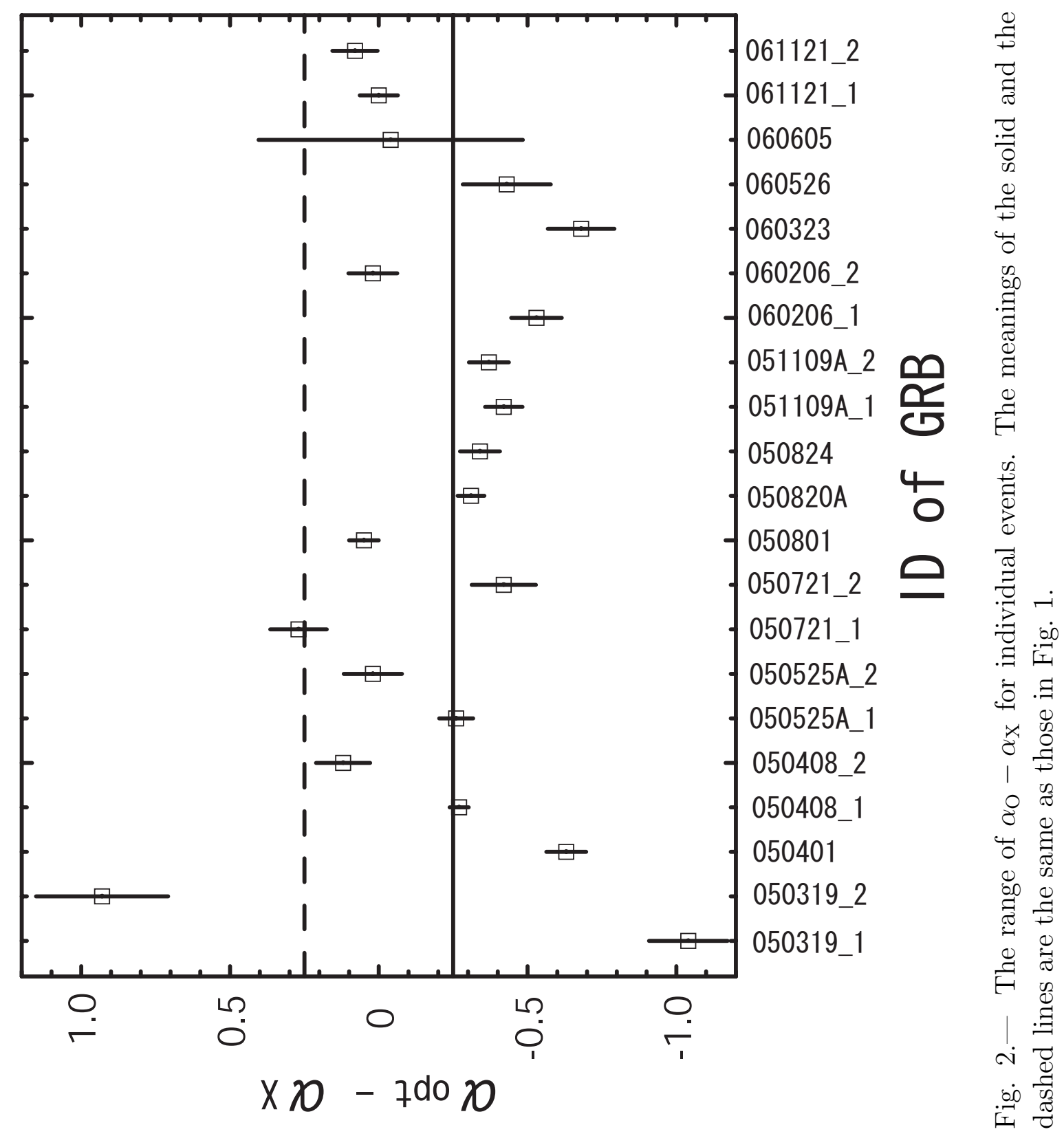


Table 1. X-ray and optical temporal decay indices during X-ray normal decay phase.

\begin{tabular}{|c|c|c|c|c|c|c|}
\hline GRB & Normal decay phase $[\mathrm{sec}]^{\mathrm{a}}$ & Optical period $[\mathrm{sec}]^{\mathrm{b}}$ & $\alpha_{\mathrm{X}}$ & $\alpha_{\mathrm{O}}$ & $\alpha_{\mathrm{O}}-\alpha_{\mathrm{X}}$ & References $^{\mathrm{c}}$ \\
\hline 050319_1 & $4.8 \times 10^{4}-2.0 \times 10^{6}$ & $1.3 \times 10^{5}-4.1 \times 10^{5}$ & $1.52 \pm 0.13$ & $0.48 \pm 0.02$ & $-1.04 \pm 0.13$ & (1) \\
\hline 050319_2 & $4.8 \times 10^{4}-2.0 \times 10^{6}$ & $4.1 \times 10^{5}-9.9 \times 10^{5}$ & $1.52 \pm 0.13$ & $2.45 \pm 0.18$ & $0.93 \pm 0.22$ & (1) \\
\hline 050401 & $3.4 \times 10^{3}-6.3 \times 10^{5}$ & $3.5 \times 10^{3}-1.4 \times 10^{5}$ & $1.39 \pm 0.06$ & $0.76 \pm 0.03$ & $-0.63 \pm 0.07$ & (2) \\
\hline 050408_1 & $2.6 \times 10^{3}-3.2 \times 10^{6}$ & $3.4 \times 10^{3}-4.6 \times 10^{4}$ & $0.86 \pm 0.01$ & $0.59 \pm 0.03$ & $-0.27 \pm 0.03$ & $(1),(3)$ \\
\hline 050408_2 & $2.6 \times 10^{3}-3.2 \times 10^{6}$ & $6.2 \times 10^{4}-3.0 \times 10^{5}$ & $0.86 \pm 0.01$ & $0.98 \pm 0.09$ & $0.12 \pm 0.09$ & $(1),(3)$ \\
\hline 050525A_1 & $3.1 \times 10^{3}-2.7 \times 10^{6}$ & $3.1 \times 10^{3}-5.7 \times 10^{4}$ & $1.51 \pm 0.04$ & $1.25 \pm 0.04$ & $-0.26 \pm 0.06$ & $(1),(4),(5)$ \\
\hline 050525A_2 & $3.1 \times 10^{3}-2.7 \times 10^{6}$ & $6.3 \times 10^{4}-4.6 \times 10^{5}$ & $1.51 \pm 0.04$ & $1.53 \pm 0.09$ & $0.02 \pm 0.10$ & $(1),(4),(5)$ \\
\hline 050721_1 & $2.3 \times 10^{3}-3.4 \times 10^{6}$ & $2.3 \times 10^{3}-7.9 \times 10^{3}$ & $0.96 \pm 0.09$ & $1.23 \pm 0.03$ & $0.27 \pm 0.09$ & (6) \\
\hline 050721_2 & $2.3 \times 10^{3}-3.4 \times 10^{6}$ & $7.9 \times 10^{3}-2.5 \times 10^{5}$ & $0.96 \pm 0.09$ & $0.54 \pm 0.06$ & $-0.42 \pm 0.11$ & (6) \\
\hline 050801 & $6.5 \times 10^{2}-3.0 \times 10^{5}$ & $7.2 \times 10^{2}-9.5 \times 10^{3}$ & $0.99 \pm 0.04$ & $1.04 \pm 0.03$ & $0.05 \pm 0.05$ & (7) \\
\hline 050820A & $2.8 \times 10^{3}-4.0 \times 10^{4}$ & $3.4 \times 10^{3}-2.0 \times 10^{4}$ & $1.04 \pm 0.02$ & $0.73 \pm 0.04$ & $-0.31 \pm 0.04$ & (8) \\
\hline 050824 & $5.9 \times 10^{4}-2.0 \times 10^{6}$ & $8.0 \times 10^{4}-4.5 \times 10^{5}$ & $0.85 \pm 0.06$ & $0.51 \pm 0.03$ & $-0.34 \pm 0.07$ & (9) \\
\hline 051109A_1 & $1.6 \times 10^{3}-5.2 \times 10^{4}$ & $1.6 \times 10^{3}-1.3 \times 10^{4}$ & $1.08 \pm 0.02$ & $0.66 \pm 0.06$ & $-0.42 \pm 0.06$ & (10) \\
\hline 051109A_2 & $5.2 \times 10^{4}-1.4 \times 10^{6}$ & $9.0 \times 10^{4}-1.0 \times 10^{6}$ & $1.35 \pm 0.03$ & $0.98 \pm 0.06$ & $-0.37 \pm 0.07$ & (10) \\
\hline 060206_1 & $2.3 \times 10^{4}-5.4 \times 10^{5}$ & $2.3 \times 10^{4}-2.5 \times 10^{4}$ & $1.39 \pm 0.08$ & $0.86 \pm 0.03$ & $-0.53 \pm 0.09$ & $(1),(11),(12)$ \\
\hline 060206_2 & $2.3 \times 10^{4}-5.4 \times 10^{5}$ & $2.5 \times 10^{4}-2.0 \times 10^{5}$ & $1.39 \pm 0.08$ & $1.41 \pm 0.02$ & $0.02 \pm 0.08$ & $(1),(11),(12)$ \\
\hline 060323 & $1.1 \times 10^{3}-2.1 \times 10^{5}$ & $1.2 \times 10^{3}-3.0 \times 10^{3}$ & $1.38 \pm 0.10$ & $0.70 \pm 0.05$ & $-0.68 \pm 0.11$ & (1) \\
\hline 060526 & $1.8 \times 10^{4}-4.2 \times 10^{5}$ & $2.0 \times 10^{4}-3.2 \times 10^{4}$ & $1.60 \pm 0.13$ & $1.17 \pm 0.07$ & $-0.43 \pm 0.15$ & (13) \\
\hline 060605 & $5.2 \times 10^{3}-2.7 \times 10^{4}$ & $2.0 \times 10^{4}-2.3 \times 10^{4}$ & $1.45 \pm 0.06$ & $1.41 \pm 0.44$ & $-0.04 \pm 0.44$ & (1) \\
\hline 061121_1 & $2.1 \times 10^{3}-1.7 \times 10^{4}$ & $4.7 \times 10^{3}-1.5 \times 10^{4}$ & $0.98 \pm 0.04$ & $0.98 \pm 0.05$ & $0.00 \pm 0.06$ & (14) \\
\hline 061121_2 & $1.7 \times 10^{4}-3.5 \times 10^{5}$ & $7.2 \times 10^{4}-3.3 \times 10^{5}$ & $1.46 \pm 0.03$ & $1.54 \pm 0.07$ & $0.08 \pm 0.08$ & $(14),(15)$ \\
\hline
\end{tabular}

a The nomal decay phase is identified in the Swift XRT data. The value of $\alpha_{\mathrm{X}}$ is determined in this period. Time zero is taken as the burst trigger time.

${ }^{\mathrm{b}}$ The period when the optical data was taken during the normal decay phase. The value of $\alpha_{\mathrm{O}}$ is determined in this epoch.

${ }^{\mathrm{c}}$ References for optical data. (1) EAFON (for specific individual events, e.g. Huang et al. (2007), Deng et al. (2007)); (2) De Pasquale et al. (2006b); (3) de Ugarte Postigo et al. (2007); (4) Klotz et al. (2005); (5) Della Valle et al. (2006); (6) Antonelli et al. (2006); (7) Rykoff et al. (2006); (8) Cenko et al. (2006); (9) Sollerman et al. (2007); (10) Yost et al. (2007); (11) Woźniak et al. (2006); (12) Stanek et al. (2007); (13) Dai et al. (2007); (14) Uemura et al. (2007); (15) Halpern et al. (2007) 
Table 2. Spectral and decay indices $\left(F_{\nu} \propto t^{-\alpha} \nu^{-\beta}\right)$ predicted by the classical external shock model.

\begin{tabular}{ccccc}
\hline \hline & $1<p<2$ & \multicolumn{2}{c}{$2<p$} & \\
& $\alpha$ & $\beta$ & $\alpha$ & $\beta$ \\
\hline$\nu<\nu_{\mathrm{m}}$ & $\frac{(4 s-3) p-2(s+3)}{3(p-1)(8-2 s)}$ & $-\frac{1}{3}$ & $\frac{s-2}{4-s}$ & $-\frac{1}{3}$ \\
$\nu_{\mathrm{m}}<\nu<\nu_{\mathrm{c}}$ & $\frac{(3-s) p+(6+s)}{2(8-2 s)}$ & $\frac{p-1}{2}$ & $\frac{3 p-1}{4}+\frac{s}{8-2 s}$ & $\frac{p-1}{2}$ \\
$\nu_{\mathrm{c}}<\nu$ & $\frac{(3-s) p+2(5-s)}{2(8-2 s)}$ & $\frac{p}{2}$ & $\frac{3 p-2}{4}$ & $\frac{p}{2}$ \\
\hline
\end{tabular}

${ }^{1}$ Note: For the case of the spherical expansion, slow cooling, and the ambient density profile given by $n \propto r^{-s}$, where $0<s<3$. The break frequency $\nu_{\mathrm{m}}$ evolves with time as $\nu_{\mathrm{m}} \propto t^{-3 / 2}$ for $2<p$ while $\nu_{\mathrm{m}} \propto t^{-\frac{(3-s) p+6-s}{(p-1)(8-2 s)}}$ for $1<p<2$, and $\nu_{\mathrm{c}}$ scales as $\nu_{\mathrm{c}} \propto t^{\frac{3 s-4}{8-2 s}}$ regardless of $p$. 